\title{
Habilidades sociais e fatores de risco e proteção na adultez emergente
}

\author{
Anderson Siqueira Pereira \\ Luciana Dutra-Thomé \\ Silvia Helena Koller \\ Universidade Federal do Rio Grande do Sul
}

\begin{abstract}
Resumo
Este estudo objetivou avaliar relações entre habilidades sociais e pensamentos negativos com fatores de proteção e de risco na adultez emergente. Participaram 521 adultos entre 18 e 30 anos $(M=22,68 ; D P=3,21)$. Os participantes foram agrupados com base em seus escores de habilidades sociais e presença de pensamentos negativos frente a interações sociais. Foram desenvolvidas ANOVAS para comparação das médias entre os grupos. Comparações de grupos demonstraram diferenças significativas entre eles, em que o grupo socialmente competente apresentou medias superiores de fatores de proteção e inferiores nos fatores de risco em relação aos demais. Habilidades sociais podem ser consideradas fator de proteção, sua presença está associada a melhores índices de autoestima, autoeficácia e qualidade nos relacionamentos.
\end{abstract}

Palavras-chave: Habilidades sociais; Fatores de risco e de proteção; Pensamentos negativos; Adultez emergente.

\section{Social skills and risk and protective factors in emerging adulthood}

\begin{abstract}
This study aimed to evaluate the relationship between social skills, negative thoughts and protective and risk factors. The study included 521 emerging adults aged between 18 and 30 years $(M=22.68$ years, $S D=3.21)$. To evaluate the relationship between variables, the participants were grouped based on their scores of social skills and presence of negative thoughts regarding social interaction. ANOVAS were conducted to compare means among groups. Results pointed out that the most socially skilled group had a greater presence of protective factors and lower of risk factors in relation to other groups. Social skills may be considered as a protective factor because they are related to increased self-esteem, self-efficacy and quality in relationships, helping in the formation of supportive networks.
\end{abstract}

Keywords: Social skills; Risk and protective factors; Support network; Emerging adulthood.

\section{Habilidades sociales y factores de riesgo y de protección en la adultez emergente}

\section{Resumen}

Este estudio tuvo como objetivo evaluar las relaciones entre las habilidades sociales y los pensamientos negativos con factores protectores y de riesgo en la adultez emergente. Participaron 521 adultos entre 18 y 30 años $(\mathrm{M}=22.68, \mathrm{SD}=3.21)$. Los participantes fueron agruparon en base a sus puntuaciones de las habilidades sociales y la presencia de pensamientos negativos en las interacciones sociales. ANOVAs fueron desarrollados para comparar las medias entre los grupos. Comparaciones de grupo mostraron diferencias significativas entre ellos, el grupo socialmente competente tuve mayores promedios de los factores de protección y más bajos en los factores de riesgo en relación con los demás. Las habilidades sociales pueden considerarse como un factor protector, su presencia se asocia con mejores niveles de autoestima, la autoeficacia y calidad en las relaciones.

Palabras clave: Habilidades sociales; Factores de riesgo y de protección; Pensamientos negativos; Adultez emergente. 


\section{Introdução}

O comportamento social dos indivíduos é composto pelo repertório de habilidades sociais e pelos pensamentos, por meio dos quais a pessoa interpreta os acontecimentos sociais em que esta inserida. É a partir destas duas variáveis que as pessoas interpretam os contextos sociais nos quais interagem e modulam seus comportamentos. Para lidar com as demandas sociais destes contextos, é necessário um repertório adequado de habilidades sociais (Caballo, 2003). Ao longo do ciclo vital, mudanças ocorrem e novas demandas sociais surgem. Na transição da adolescência para a vida adulta, jovens precisam utilizar um repertório de habilidades sociais mais complexo para ter um desempenho social adequado. Esta fase é caracterizada por uma série de desafios impostos pelos novos contextos sociais aos quais eles serão apresentados, em destaque para o laboral/educacional e familiar (Pereira, 2015). A fim de destacar a importância das habilidades sociais na transição para a vida adulta, o presente estudo objetivou avaliar as relações das habilidades sociais e de pensamentos negativos referentes às interações sociais com variáveis intrínsecas (autoestima, autoeficácia e ansiedade social) e extrínsecas (relacionamentos sociais e violência), consideradas protetivas ou de risco.

Habilidades sociais podem ser compreendidas como uma classe de comportamentos específicos para lidar, resolver e evitar problemas nas relações interpessoais (Caballo, 2003). Elas são responsáveis pela manutenção dos relacionamentos sociais, contribuindo para a aceitação do grupo no qual se pretende ingressar ou manter (Gresham, 2009). São exemplos de habilidades sociais: iniciar e manter conversações; pedir ajuda; dar e receber elogios; expressar agrado e desagrado; fazer e responder perguntas; expressar sentimentos; lidar com críticas; fazer e recusar pedidos; escutar empaticamente (Caballo, 2003). Além destas, podem ser incluídos aspectos não verbais ligados às habilidades sociais, como postura, contato visual, gestos, aparência e entonação de voz (Del Prette \& Del Prette, 1999).

Além do componente comportamental, o desempenho social das pessoas é mediado pela forma como as interações sociais são interpretadas cognitivamente. Para um desempenho social adequado, a pessoa deve avaliar o contexto social de forma clara, observar e entender as demandas sociais, e escolher os melhores comportamentos em seu repertório de habilidades sociais. Porém, quando as relações interpessoais são avaliadas de forma errônea, pode ocorrer uma escolha não adequada de comportamentos, o que geralmente leva a consequências desagradáveis. Isso é particularmente problemático nos casos das pessoas que desenvolvem pensamentos negativos em avaliações nem sempre realistas das interações sociais, pois elas apresentam maior dificuldade em apresentar desempenhos sociais satisfatórios. Ademais, os pensamentos com interpretação negativa tendem a desencadear sintomas de ansiedade, os quais dificultam a interação com outras pessoas (Caballo, 2003). Desta forma, pensamentos negativos frente às relações sociais podem ser definidos como interpretações negativas das situações em si, do próprio desempenho ou da avaliação dos demais em relação ao desempenho social da pessoa. Alguns exemplos seriam: medo de avaliação negativa dos demais, de expressar opiniões contrárias, de fazer "papel de bobo" ou de inadequação. Estes tipos de pensamentos são comuns em pessoas socialmente ansiosas, o que interfere em sua competência social e pode resultar em isolamento social (Caballo, 2003; Wagner, Pereira, \& Oliveira, 2014). Além disso, a presença destes pensamentos é um dos principais sintomas do Transtorno de Ansiedade Social (American Psychiatric Association - APA, 2014).

Ter um repertório adequado de habilidades sociais é fundamental na transição para a vida adulta, especialmente numa época em que os jovens estão prolongando a passagem para adultez em países industrializados ou recentemente industrializados. Diz-se que entre a adolescência e a adultez surgiu uma nova fase desenvolvimental, chamada de adultez emergente, resultado do comportamento dos jovens de postergar a assunção de papéis adultos como o casamento, a independência financeira dos pais e a constituição de uma família (Arnet, 2011). O surgimento desta nova fase se dá por uma série de mudanças na sociedade, como a entrada da mulher no mercado de trabalho, a flexibilização dos padrões de sexualidade, e a necessidade dos jovens terem mais anos de estudo e treinamento para entrar em um mercado de trabalho cada vez mais exigente (Arnett, 2011; Dutra-Thomé \& Koller, 2014a). A característica subjetiva marcante da adultez emergente tem sido apontada como o sentimento de ambivalência, pois os jovens não se sentem adolescentes nem adultos, o que ocorre justamente por estarem vivendo um período alongado de exploração de suas identidades e não assunção de compromissos definitivos em longo prazo. Diante das incertezas que enfrentam, vivenciam também sentimentos de negatividade e instabilidade, o que pode explicar o motivo pelo qual a adultez emergente tem sido apontada como um período em que indivíduos se envolvem em situações de risco, como uso de drogas e sexo desprotegido. 
No Brasil, as pesquisas de Dutra-Thomé e Koller se destacam por tentar caracterizar esta nova fase nos jovens brasileiros (Dutra-Thomé \& Koller, 2014a; 2014b). Em um de seus estudos (Dutra-Thomé \& Koller, 2014b) foi identificado que os jovens se percebem ambivalentes entre ser ou não adulto, porém jovens de nível socioeconômico alto têm sido apontados como mais ambivalentes do que jovens de nível socioeconômico baixo. Isso ocorre pelo fato de que os jovens de nível socioeconômico baixo tendem a assumir papeis de adultos precocemente, o que os leva a se perceberem como adultos mais cedo, diante das responsabilidades que assumem e menor oportunidade de experimentar um período prolongado de exploração da identidade.

A adultez emergente é um período crítico para jovens de ambos os níveis socioeconômicos (Arnett, 2011). Diversos são os problemas que podem surgir na vida dos jovens. Por exemplo, no campo laboral, eles enfrentam um mercado de trabalho competitivo, flexível e que lhes exige níveis de preparo educacional e profissional altíssimos para conseguirem um trabalho decente (Antunes, 2011; Blustein, 2006). Ou então, tendo em vista que, atualmente, aos jovens é permitida a possibilidade de viver diversas experiências amorosas antes de optarem por uma relação mais estável, a busca de um parceiro amoroso ideal pode gerar frustrações (Arnett, 2011). A presença de fatores de proteção pode amenizar os efeitos dos eventos negativos e dos desafios enfrentados pelos jovens. Fatores de proteção são características intrínsecas (como autoestima e autoeficácia) ou extrínsecas (como a relação com amigos, familiares, rede de apoio) que a fortalecem e lhe dão suporte para lidar com situações problema. Estes fatores não atuam isoladamente, mas interagem entre si para auxiliar na alteração do comportamento da pessoa, desenvolvendo uma experiência de proteção às situações de risco e auxiliando na solução dos problemas (Poletto \& Koller, 2008). Justamente pela adultez emergente ser um período em que jovens estabelecem uma série de interações sociais que ocupam uma parcela considerável do seu tempo (ex.: círculos de amizade, trabalho e estudo), entende-se que esses círculos podem funcionar como redes de apoio em potencial para ajudar os jovens a lidar com dificuldades que possam surgir em suas vidas. Para isso, é necessário que os jovens apresentem um repertório de habilidades sociais que os auxiliem a construir e manter relacionamentos sociais que se tornem redes de apoio efetivas.

Além dos fatores de proteção, os jovens são expostos a fatores de risco. Os fatores de risco constituem eventos e características negativas da vida das pessoas, e sua presença aumenta as chances de problemas físicos, emocionais e sociais se manifestarem (Poletto \& Koller, 2008). São exemplos de fatores de risco: família disfuncional, instabilidade econômica, experiências de violência física/sexual, vivência em comunidades violentas e locais de trabalho insalubres (Pesce, Assis, Santos, Oliveira, \& Cruz, 2004; Poletto \& Koller, 2008). Da mesma forma, certos comportamentos tais como uso de drogas e sexo desprotegido também podem colocar em risco o desenvolvimento destes jovens. Estes fatores tendem a aumentar a vulnerabilidade do indivíduo a situações adversas na vida.

Deve-se levar em conta que cada pessoa pode reagir de maneira diferente a fatores de proteção e de risco. Portanto, não é apenas a presença destes fatores que define seu impacto na vida do indivíduo, mas também a intensidade, a frequência e a maneira que a pessoa os interpreta. Sabendo-se que durante a adultez emergente os jovens enfrentam diversas dificuldades, e que a maneira como os jovens lidam com as dificuldades da vida interfere no impacto das mesmas, cabe considerar o conceito de resiliência. Ele pode ser definido como a capacidade da pessoa conseguir se adaptar e superar as situações da vida da melhor forma, conseguindo manter a saúde mental (Pesce, Assis, Santos, Oliveira, \& Cruz, 2004). O processo de resiliência é complexo, sendo influenciado por uma gama de fatores. Desta forma, ao se analisar a forma como a pessoa irá lidar com os problemas que surgem em sua vida, é necessário levar em conta a interação dos fatores de risco e proteção. Quando a presença de fatores de proteção é suficiente para amenizar os riscos, a pessoa possuirá recursos para melhor lidar com os eventos estressores e conseguir desfechos positivos para as situações. Por outro lado, na falta de fatores de proteção e com a presença de fatores de risco, a pessoa terá menos recursos, o que aumentará a chances de desfechos negativos, e a deixará vulnerável a problemas sociais e emocionais (ex.: uso de drogas, isolamento, desemprego, depressão).

Considerando o exposto, o presente estudo avaliou as habilidades sociais e a presença de pensamentos negativos referentes às interações sociais em adultos emergente brasileiros, e suas relações com variáveis de interação social consideradas protetivas (relação familiar, relacionamentos de amizade, autoestima e autoeficácia); ou de risco (ansiedade social, violência familiar e comunitária). O objetivo foi avaliar se grupos com os níveis diferentes de habilidades sociais e de presença de pensamentos negativos apresentavam médias diferentes nos fatores de proteção (autoestima, autoeficácia, satisfação com o trabalho, relacionamentos familiar e de amizade) e de risco (ex.: ansiedade social, violência familiar e comunitária). 


\section{Método}

\section{Amostra}

Participaram do estudo 521 indivíduos. Os critérios de inclusão foram: idade entre 18 e 30 anos, residir no Brasil e ter respondido de forma correta as perguntas controle incluídas ao longo do questionário. Estas perguntas controle solicitavam ao participante marcar uma alternativa específica, no intuito de ter certeza que as questões não haviam sido marcadas aleatoriamente ou sem atenção. A amostra foi predominantemente feminina $(n=344,66 \%)$, com idade média de 22,68 $\operatorname{anos}(\mathrm{DP}=3,21)$. Os demais dados sociodemográficos podem ser observados na Tabela 1.

TABELA 1

Dados Sociodemográficos da amostra de jovens adultos $(\mathrm{N}=521)$

\begin{tabular}{|c|c|c|}
\hline & $n$ & $\%$ \\
\hline \multicolumn{3}{|l|}{ Sexo } \\
\hline Masculino & 177 & 44 \\
\hline Feminino & 344 & 66 \\
\hline \multicolumn{3}{|l|}{ Estado Civil } \\
\hline Solteiro & 451 & 86,6 \\
\hline Casado & 23 & 4,4 \\
\hline Mora Junto & 29 & 5,6 \\
\hline Separado/divorciado & 3 & 0,6 \\
\hline Outro & 15 & 2,9 \\
\hline \multicolumn{3}{|l|}{ Escolaridade } \\
\hline \multicolumn{3}{|c|}{ Fundamental incompleto } \\
\hline Médio incompleto & 2 & 0,4 \\
\hline Médio completo & 20 & 3,8 \\
\hline Técnico incompleto & 2 & 0,4 \\
\hline Técnico completo & 4 & 0,8 \\
\hline Superior incompleto & 365 & 70,1 \\
\hline Superior completo & 75 & 14,4 \\
\hline PósGraduação & 53 & 10,2 \\
\hline \multicolumn{3}{|l|}{ Regiões } \\
\hline Norte & 31 & 6 \\
\hline Nordeste & 88 & 16,9 \\
\hline Centro-Oeste & 58 & 11,2 \\
\hline Sudeste & 156 & 30,1 \\
\hline Sul & 188 & 36,1 \\
\hline
\end{tabular}

$\mathrm{n}=$ Amostra.

\section{Instrumentos}

Escala Multidimensional de Expressão Social (EMES-C e EMES-M): As EMES-M e EMES-C foram desenvolvidas por Caballo (2003) e tem por objetivo avaliar as habilidades sociais e os pensamentos negativos frente à interação social, respectivamente. Foram adaptadas para o português brasileiro por Pereira (2015). A EMES-M é composta por 64 itens, apresentando em sua validação brasileira uma estrutura de 12 fatores e alfa de Cronbach da escala total de 0,950 (Pereira, 2015). A EMES-C é composta por 44 itens e apresenta uma estrutura de oito fatores, com alfa de Cronbach da escala total de 0,954 (Pereira, 2015). Neste estudo, foram usadas as escalas totais de ambas as medidas.

Dimensional Anxiety Scale - Social Anxiety Disorder (SAD-D; APA, 2014): medida dimensional breve de autorrelato para diagnosticar sintomas de ansiedade social. A escala solicita ao respondente que avalie, numa escala de cinco pontos ( 0 - Nunca a 5 - O tempo todo) e em relação aos últimos sete dias, o quanto as afirmativas representam seus sintomas físicos e cognitivos de ansiedade; bem como seus comportamentos frente a situações aversivas. $\mathrm{O}$ instrumento se mostra promissor, diante de sua atualidade e evidências de confiabilidade em contextos comunitários e clínicos tanto na versão original (Lebeau et al., 2012) quanto na adaptada para ao alemão (Beesdo-Baum et al., 2012). A SAD-D foi adaptada e validada para o português brasileiro (DeSousa et al., 2016), apresentando alfa de Cronbach de 0,899 .

Questionário Juventude Brasileira 2 2 a versão (Dell'Aglio, Koller, \& Cerqueira-Santos, 2011): investiga questões sobre educação, saúde, trabalho, comportamentos de risco (ex.: drogas, suicídio, sexualidade, violência), fatores de risco (ex.: violência intrafamiliar e extrafamiliar, deficiência, conflito com a lei, eventos estressores, preconceito) e fatores protetores sociais (ex.: lazer, rede de apoio) e pessoais (ex.: espiritualidade, autoestima, auto eficácia, perspectivas para o futuro). É composto por 77 questões, sendo algumas de múltipla escolha e outras em escalas de intensidade e frequência. Para as análises deste estudo, foram utilizadas as questões de levantamento sociodemográfico, o Inventário de Autoeficácia Geral Percebida (Sbicigo, Teixeira, Dias, \& Dell'Aglio, 2012), o Inventário de Autoestima de Rosenberg (Hutz \& Zanon, 2011), as questões referentes a relação familiar, violência familiar e comunitária.

Questionário para avaliação das relações de amizade: baseado nos questionários de MCGill para avaliar qualidade da amizade (Souza \& Hutz, 2007) tem por objetivo avaliar a quantidade e qualidade das relações de amizade. É composto de três 
questões abertas e por 30 itens que apresentam frases sobre a impressão que o respondente possui de suas amizades. Os itens são pontuados em uma escala de cinco pontos ( 1 - Discordo totalmente a 5 - Concordo totalmente). A consistência interna da escala foi avaliada para esta amostra, apresentando um alfa de Cronbach de 0,97.

Escala de satisfação no trabalho do OSI (Occupational Stress Indicator; Swan, De Moraes, \& Coopes, 1993): É composta por 22 questões sobre características do ambiente de trabalho, as quais devem ser pontuadas em uma escala de seis pontos, variando de 1 (enorme insatisfação) a 6 (enorme satisfação). A consistência interna neste estudo foi de 0,942 .

\section{Procedimentos}

Os instrumentos foram agrupados em um protocolo online a partir da plataforma SurveyMonkey. A divulgação da pesquisa foi realizada em redes sociais, páginas de universidades e indicação de outras pessoas pelos participantes. A pesquisa foi aprovada pelo parecer número 636.992/2014 do Comitê de Ética do Instituto de Psicologia da UFRGS, sendo que todos os participantes concordaram com o Termo de Consentimento Livre e Esclarecido (TCLE).

\section{Análise de Dados}

Foram realizadas correlações de Pearson e testes $t$ de Student entre variáveis sociodemográficas idade e nível educacional e as médias dos escores totais da EMES-M e EMES-C. Como as escalas EMES-M e EMES C não possuem pontos de corte, foram calculados os tercis a partir dos escores totais de ambas as escalas para composição de três grupos. Os grupos foram compostos pelo o mesmo número de participantes baseados, e representam três níveis de habilidades sociais e pensamentos negativos, a partir das médias amostrais: nível baixo, médio e alto. Com esta divisão, foram realizadas ANOVAs para comparar as médias entre os grupos nas variáveis avaliadas neste estudo. Para realização das análises com os dados relacionados à satisfação no trabalho, foi utilizada apenas a subamostra que respondeu estar trabalhando $(n=174)$.

\section{Resultados}

Inicialmente foram analisadas se as variáveis presentes no estudo respeitavam o critério de normalidade para o uso de estatísticas paramétricas. Os resultados indicaram que este critério foi respeitado. Após, foram realizadas análises de correlação utilizando os níveis de habilidades sociais medidos pela EMES-C e EMES-M, a idade dos participantes e o nível educacional dos respondentes e de seus pais. Não houve correlações estatisticamente significativas. Foi realizado um teste $t$ em dois grupos por idade (grupo $\mathrm{a}=18$ aos 24 ; grupo $\mathrm{b}=25$ aos 30 ) e por sexo. O teste $t$ demonstrou que o grupo $\mathrm{b}(\mathrm{M}=180,12 ; \mathrm{DP}=34,29)$ apresentou médias superiores nos escores de habilidades sociais em relação ao grupo a $(\mathrm{M}=170,88$; $\mathrm{DP}=32,84 ; t=2,551 ; d f=518, p=0,011)$. Em relação aos pensamentos negativos, o Grupo a $(\mathrm{M}=132,46$, $\mathrm{DP}=32,84)$ apresentou médias superiores que o Grupo b $(\mathrm{M}=125,59 ; \mathrm{DP}=31,52 ; t=-2,167 ; d f=519$; $p=0,031)$. Em relação ao sexo, não houve diferenças significativas.

$\mathrm{Na}$ sequência das análises, os participantes foram divididos em três grupos a partir de seus escores gerais nas escalas EMES-C e EMES-M (GAh: Grupo com médias mais altas de habilidades sociais; GMh: Grupo com médias medianas e; GBh: Grupo com médias baixas); e três grupos referentes à presença de pensamentos negativos (GAp: Grupo com alta presença de pensamentos negativos; GMp: Grupo com valores medianos e; GBp: Grupo com baixa presença). Esta divisão foi realizada pelo fato de que as escalas não possuem pontos de corte para a identificação da amostra. Dessa forma, a divisão por tercis apontou pontos de corte a partir das médias da amostra. Estes grupos foram utilizados para a realização das ANOVAs.

Foram realizadas ANOVAs para comparar as médias das variáveis autoeficácia, autoestima, relacionamento familiar, relacionamento de amizade, satisfação no trabalho, ansiedade social, violência familiar e violência comunitária dos grupos divididos por níveis habilidades sociais e pensamentos negativos. Nos grupos divididos pelos níveis de habilidade social foram encontradas diferenças estatisticamente significativas nas médias de escore de relacionamento familiar e de amizade, ansiedade social, autoestima e autoeficácia. As variáveis autoestima, autoeficácia e ansiedade social apresentaram diferenças entre os três grupos, enquanto as variáveis relacionamento de amizade e familiar apresentaram diferenças entre o grupo GAh e os demais. Na variável satisfação com o trabalho houve diferença estatisticamente significativa apenas entre as médias do grupo GAh e o GBh. O grupo com maior escore de habilidades sociais (GAh) apresentou médias mais elevadas de satisfação no trabalho, como pode ser observado na Tabela 2. 
TABELA 2

Resultado das ANOVAs entre os grupos com altos (GAh), médios (GMh) e baixos (GBh) níveis de habilidades sociais (n=521)

\begin{tabular}{|c|c|c|c|c|c|c|c|c|}
\hline \multirow{2}{*}{ Variáveis } & \multicolumn{3}{|c|}{ Média Grupos (DP) } & \multirow{2}{*}{$F$} & \multirow{2}{*}{$p$} & \multicolumn{2}{|c|}{$\begin{array}{c}\text { Testes Post hoc } \\
\text { Bonferroni }\end{array}$} & \multirow{2}{*}{ Cohen's d } \\
\hline & GAh & $G M h$ & $G B h$ & & & Comparação & $p$ & \\
\hline \multirow[t]{3}{*}{ Amizade } & 134,20 & 128,13 & 124,38 & 11,10 & $<0,001$ & GAh vs. GMh & 0,013 & 0,324 \\
\hline & $(18,76)$ & $(18,69)$ & $(21,28)$ & & & GAh vs. GBh & $<0,001$ & 0,490 \\
\hline & & & & & & GMh vs. GBh & 0,226 & 0,187 \\
\hline \multirow[t]{3}{*}{ Ansiedade Social } & 18,12 & 24,27 & 31,07 & 97,59 & $<0,001$ & GAh vs. GMh & $<0,001$ & 0,792 \\
\hline & $(6,00)$ & $(9,19)$ & $(10,15)$ & & & GAh vs. GBh & $<0,001$ & 1.553 \\
\hline & & & & & & GMh vs. GBh & $<0,001$ & 0,702 \\
\hline \multirow[t]{3}{*}{ Autoeficácia } & 36,05 & 31,47 & 27,96 & 81,81 & $<0,001$ & GAh vs. GMh & $<0,001$ & 0,805 \\
\hline & $(5,74)$ & $(5,64)$ & $(6,14)$ & & & GAh vs. GBh & $<0,001$ & 1,361 \\
\hline & & & & & & GMh vs. GBh & $<0,001$ & 0,595 \\
\hline \multirow[t]{3}{*}{ Autoestima } & 41,01 & 34,63 & 28,86 & 110,03 & $<0,001$ & GAh vs. GMh & $<0,001$ & 0,879 \\
\hline & $(7,28)$ & $(7,24)$ & $(8,31)$ & & & GAh vs. GBh & $<0,001$ & 1,555 \\
\hline & & & & & & GMh vs. GBh & $<0,001$ & 0,740 \\
\hline \multirow{3}{*}{$\begin{array}{l}\text { Satisfação no trabalho } \\
(n=174)^{*}\end{array}$} & 90,38 & 87,22 & 78,90 & 4,80 & 0,009 & GAh vs. GMh & 1,000 & 0,149 \\
\hline & $(21,57)$ & $(20,92)$ & $(18,21)$ & & & GAh vs. GBh & 0,008 & 0.575 \\
\hline & & & & & & GMh vs. GBh & 0,112 & 0.424 \\
\hline \multirow[t]{3}{*}{ Relação familiar } & 56,42 & 52,66 & 50,83 & 9,55 & $<0,001$ & GAh vs. GMh & 0,013 & 0,315 \\
\hline & $(11,53)$ & $(12,30)$ & $(12,59)$ & & & GAh vs. GBh & $<0,001$ & 0.463 \\
\hline & & & & & & GMh vs. GBh & 0,482 & 0.147 \\
\hline \multirow[t]{3}{*}{ Violência Familiar } & 1,36 & 1,63 & 1,53 & 1,88 & 0,153 & GAh vs. GMh & 0,166 & 0.212 \\
\hline & $(1,20)$ & $(1,34)$ & $(1,26)$ & & & GAh vs. GBh & 0,702 & 0,138 \\
\hline & & & & & & GMh vs. GBh & 1,000 & 0,077 \\
\hline \multirow[t]{3}{*}{ Violência Comunitária } & 0,91 & 0,99 & 1,15 & 2,82 & 0,061 & GAh vs. GMh & 1,000 & 0,082 \\
\hline & $(0,92)$ & $(1,02)$ & $(0,93)$ & & & GAh vs. GBh & 0,060 & 0,259 \\
\hline & & & & & & GMh vs. GBh & 0,369 & 0,164 \\
\hline
\end{tabular}

* Análise realizada apenas com subamostra de participantes que estavam trabalhando no momento da pesquisa; ANOVA = Analysis of Variance;

Nas análises com os grupos divididos pela presença de pensamentos negativos houve diferenças estatisticamente significativas nas médias de escore nas variáveis violência comunitária e familiar, ansiedade social, autoestima, autoeficácia e dos relacionamentos familiares e de amizade. As variáveis autoestima, autoeficácia e ansiedade social apresentaram diferenças entre os três grupos. O grupo GBp apresentou médias superiores de qualidade dos relacionamentos de amizade em relação aos demais grupos. O grupo com maior presença de pensamentos negativos (GAp) apresentou médias superiores em relação ao grupo GBp na variável violência comunitária, e inferiores aos dois grupos na variável Relacionamento familiar. Não houve diferenças estatisticamente significativas entre os grupos quanto à satisfação no trabalho. As médias de cada variável podem ser observadas na Tabela 3. 
TABELA 3

Resultado das ANOVAs entre os grupos com alta (GAp), média (GMp) e baixa (GBp) frequência de pensamentos negativos frente a situações sociais $(n=521)$

\begin{tabular}{|c|c|c|c|c|c|c|c|c|}
\hline \multirow{2}{*}{ Variáveis } & \multicolumn{3}{|c|}{ Média Grupos (DP) } & \multirow{2}{*}{$F$} & \multirow{2}{*}{$p$} & \multicolumn{2}{|c|}{$\begin{array}{c}\text { Testes Post hoc } \\
\text { Bonferroni }\end{array}$} & \multirow{2}{*}{ Cohen's d } \\
\hline & GAp & $G M p$ & $G B p$ & & & Comparação & $p$ & \\
\hline \multirow[t]{3}{*}{ Amizade } & 124,65 & 127,05 & 134,75 & 12,92 & $<0,001$ & GAp vs. GMp & 0,774 & 0,115 \\
\hline & $(22,00)$ & $(19,53)$ & $(16,84)$ & & & GAp vs. GBp & $<0,001$ & 0,516 \\
\hline & & & & & & GMp vs. GBp & 0,001 & 0,422 \\
\hline \multirow[t]{3}{*}{ Ansiedade Social } & 32,33 & 23,29 & 17,94 & 140,77 & $<0,001$ & GAp vs. GMp & $<0,001$ & 0,999 \\
\hline & $(10,06)$ & $(7,92)$ & $(6,01)$ & & & GAp vs. GBp & $<0,001$ & 1,737 \\
\hline & & & & & & GMp vs. GBp & $<0,001$ & 0,761 \\
\hline \multirow[t]{3}{*}{ Autoeficácia } & 28,08 & 31,32 & 35,91 & 78,06 & $<0,001$ & GAp vs. GMp & $<0,001$ & 0,549 \\
\hline & $(6,13)$ & $(5,67)$ & $(5,84)$ & & & GAp vs. GBp & $<0,001$ & 1,308 \\
\hline & & & & & & GMp vs. GBp & $<0,001$ & 0,797 \\
\hline \multirow[t]{3}{*}{ Autoestima } & 28,99 & 34,80 & 40,60 & 99,22 & $<0,001$ & GAp vs. GMp & $<0,001$ & 0,729 \\
\hline & $(8,30)$ & $(7,63)$ & $(7,31)$ & & & GAp vs. GBp & $<0,001$ & 1,485 \\
\hline & & & & & & GMp vs. GBp & $<0,001$ & 0,776 \\
\hline \multirow{3}{*}{$\begin{array}{l}\text { Satisfação no trabalho } \\
(n=174)^{*}\end{array}$} & 81,04 & 84,89 & 90,01 & 2,79 & 0,064 & GAp vs. GMp & 1,000 & 0,205 \\
\hline & $(19,87)$ & $(17,62)$ & $(23,12)$ & & & GAp vs. GBp & 0,065 & 0,416 \\
\hline & & & & & & GMp vs. GBp & 0,503 & 0,249 \\
\hline \multirow[t]{3}{*}{ Relação familiar } & 49,95 & 53,94 & 56,06 & 11,62 & $<0,001$ & GAp vs. GMp & 0,007 & 0.326 \\
\hline & $(13,12)$ & $(11,32)$ & $(11,80)$ & & & GAp vs. GBp & $<0,001$ & 0.490 \\
\hline & & & & & & GMp vs. GBp & 0,315 & 0,183 \\
\hline \multirow[t]{3}{*}{ Violência Familiar } & 1,70 & 1,43 & 1,37 & 3,32 & 0,037 & GAp vs. GMp & 0,160 & 0,211 \\
\hline & $(1,31)$ & $(1,25)$ & $(1,22)$ & & & GAp vs. GBp & 0,046 & 0,261 \\
\hline & & & & & & GMp vs. GBp & 1,000 & 0,049 \\
\hline \multirow[t]{3}{*}{ Violência Comunitária } & 1,24 & 0,88 & 0,91 & 7,63 & 0,001 & GAp vs. GMp & 0,002 & 0,376 \\
\hline & $(1,02)$ & $(0,89)$ & $(0,94)$ & & & GAp vs. GBp & 0,003 & 0,336 \\
\hline & & & & & & GMp vs. GBp & 1,000 & 0,033 \\
\hline
\end{tabular}

* Análise realizada apenas com subamostra de participantes que estavam trabalhando no momento da pesquisa; ANOVA = Analysis of Variance;

\section{Discussão}

As relações sociais são importantes durante toda a vida. Entretanto, a adultez emergente é um período particularmente importante pela ampliação da rede social que os jovens vivenciam nas relações laborais, educacionais e familiares. É através destas relações que os jovens criam suas redes de apoio e contatos para se desenvolver na sociedade (Rodrigues \& Ferreira,
2012). Como visto nos resultados das análises da ANOVA, as habilidades sociais são importantes para as interações sociais, uma vez que os grupos com níveis mais altos de habilidades sociais apresentaram melhores relações com a família e os amigos, as quais podem ser consideradas formadoras de redes de apoio. Todavia, é preciso levar em conta que as habilidades sociais de todos os indivíduos envolvidos na relação são importantes para que as redes de apoio 
tenham, de fato, um papel relevante na vida dos indivíduos. No presente estudo, levaram-se em conta as habilidades sociais apenas de um dos envolvidos na relação. Porém, mesmo com esta limitação, foi possível perceber a diferença na qualidade dos relacionamentos quando comparados os diferentes níveis de habilidades sociais.

As habilidades sociais também são importantes para as relações de amizades. Conforme os resultados, os jovens com níveis mais elevados de habilidades sociais possuíam melhor qualidade das relações de amizade. Relações de amizade de qualidade são consideradas importantes para a felicidade, e constituem fontes de apoio em situações problemáticas, sendo permeadas por habilidades de escuta, empatia, apoio e autorrevelação (Demir \& Weitekamp, 2007; Fehr, 1996). Porém, para o efetivo funcionamento destas redes de apoio, é necessário que o indivíduo tenha as habilidades necessárias para que as relações sejam satisfatórias com estes grupos quanto para pedir ajuda a estas redes. Os resultados indicaram que a presença de pensamentos negativos em relação ao contato social e a ansiedade social podem prejudicar a qualidade das relações interpessoais, visto que o grupo com as maiores médias de pensamentos negativos apresentou qualidade inferior tanto nas relações de amizade, quanto nas familiares. Além disso, tanto a falta de habilidades sociais necessárias para pedir ajuda, como a presença de pensamentos negativos referentes às interações sociais podem inibir o comportamento de pedir ajuda quando necessário, dificultando ainda mais a resolução do problema enfrentado.

Observou-se também que os jovens mais velhos, quando comparados aos mais jovens, apresentaram médias superiores de habilidades sociais e inferiores de pensamentos negativos referentes às interações sociais. Este resultado vai ao encontro do que é relatado na literatura, visto que, com o passar dos anos, novas experiências e contatos sociais irão demandar do indivíduo novos comportamentos sociais aos quais ele terá que se adaptar, aumentando assim seu repertório de habilidades (Del Prette \& Del Prette, 2009). Além disso, estudos indicam que, com a idade, os jovens amadurecem cognitivamente e possuem melhores condições para tomada de decisão (Donnellan, Conger, \& Burzette, 2007; Tanner \& Arnett, 2009). Vê-se, portanto, que a adultez emergente é um período no qual os jovens podem superar histórias de exposição a fatores de risco (Masten \& Tellegen, 2012), pelas novas relações sociais que estabelecem, por possuírem um repertório mais vasto de habilidades sociais e pelo amadurecimento cognitivo. Quanto ao nível educacional, não houve correlação significativa com as habilidades sociais. Este resultado pode demonstrar que as experiências de jovens com níveis educacionais diferentes, mesmo que distintas, acabam por estimular igualmente o desenvolvimento de habilidades sociais.

Os resultados apontaram relações positivas entre as habilidades sociais e fatores de proteção intrínsecos, no caso, autoestima e autoeficácia. A autoestima pode ser definida como um conjunto de valores e crenças do indivíduo sobre seu próprio valor, sendo considerada um importante indicador de saúde mental (Sbicigo, Bandeira, \& Dell'Aglio, 2010). Ela possui ligação com os relacionamentos interpessoais, visto que a forma como a pessoa se sente em relação a si mesma também está relacionada à percepção e tratamento dos outros em relação a ela. Relacionamentos positivos com as pessoas que integram o círculo social tendem a aumentar o sentimento de pertencimento e as habilidades sociais são importantes para o início e a manutenção destas relações sociais. Da mesma forma, a crença da pessoa na sua capacidade de resolver problemas, expressa em níveis saudáveis de autoeficácia (Sbicigo, Teixeira, Dias, \& Dell'Aglio, 2012), está relacionada às interações sociais, visto que muitos dos problemas que uma pessoa enfrenta no seu dia a dia estão relacionados ao meio social. Desta forma, um bom repertório de habilidades sociais pode aumentar o senso de autoeficácia da pessoa para resolver problemas sociais, pois ele contribui para que a pessoa tenha os recursos necessários para lidar com a situação. Por outro lado, déficits nas habilidades sociais poderão diminuir a autoeficácia da pessoa, pois ela não se percebe como possuidora de um repertório adequado para lidar com as interações sociais. Isso pode resultar no aumento de sua ansiedade frente aos problemas, o que prejudicará seu desempenho, e, possivelmente, terá também impacto negativo em sua autoestima.

A ansiedade social apresentou uma relação negativa com as habilidades sociais. A ansiedade social dificulta a interação do indivíduo com os demais, limitando sua rede de contatos e impedindo que ele possa iniciar e desenvolver novos relacionamentos. Em níveis elevados, ela pode levar a pessoa a ter altos picos de ansiedade em situações sociais, gerando isolamento e aumentando as chances de desenvolver outros transtornos, como a depressão (American Psychiatric Association, APA, 2014; Segrin, 2000; Wagner, Pereira, \& Oliveira, 2014). A literatura aponta que déficits em habilidades sociais são associados à ansiedade social (Caballo, 2003; Wagner, Pereira, \& Oliveira, 2014), o que foi confirmado pelo presente estudo. Como observado nos resultados da ANOVA (Tabela 3), há diferenças estatisticamente significativas das médias da SAD-D entre os três grupos de níveis 
de habilidades sociais. A partir deste resultado, podese supor que uma intervenção voltada para o aumento das habilidades sociais pode diminuir os sintomas e o sofrimento causado pela ansiedade social.

As comparações realizadas entre grupos pela frequência de pensamentos negativos demonstraram diferenças estatisticamente significativas nas médias de escore das variáveis ansiedade social, violência familiar e comunitária. $\mathrm{O}$ resultado referente à ansiedade social era esperado, visto que a presença de pensamentos negativos referentes às situações sociais é um dos principais sintomas do transtorno de ansiedade social (APA, 2014). Tais pensamentos podem aumentar a ansiedade do indivíduo em situações nas quais exista a necessidade de interação social, o que pode prejudicar o seu desempenho social.

A associação entre a presença de pensamentos negativos e as situações de violência familiar e comunitária pode ter duas explicações. A primeira está relacionada com a forma como a pessoa vê as relações interpessoais, visto que, se ela é vítima de violência pelas pessoas que se relaciona com ela, ela acaba tendo a percepção de que toda a interação social pode ser violenta, o que a faz desenvolver crenças negativas em relação às interações sociais. Outra explicação seria a de que, com a presença dos pensamentos negativos, é difícil para a pessoa desenvolver uma rede de apoio adequada e, quando algum tipo de violência ocorre, ela não tem a quem recorrer. Isso pode levar ao isolamento da pessoa em um meio no qual este tipo de violência seja recorrente. Estas duas explicações também podem ser complementares, visto que uma situação pode causar a outra, criando um ciclo de retroalimentação da violência.

Quanto aos fatores de proteção intrínsecos, observou-se que tanto as médias de autoestima quanto as de autoeficácia apresentaram diferenças estatisticamente significativas nos três grupos referentes aos pensamentos negativos. Esse resultado demonstra o quanto os pensamentos negativos podem ter um impacto negativo na forma como a pessoa se percebe. Pessoas com níveis elevados de pensamentos de inadequação são mais propensas a terem baixa autoestima, uma vez que os pensamentos negativos afetam as interações que estabelecem, bem como a percepção de si mesmas. Estes pensamentos poderão atrapalhar o desempenho social, diminuindo assim a autoeficácia social, que diminuirá ainda mais a autoestima, reiniciando o ciclo.

Dentro do ambiente de trabalho, as habilidades sociais possuem tanto o papel de mediar às relações com colegas e superiores, quanto o de auxiliar na execução das atividades da própria profissão (Bolsoni-
Silva, 2002; Del Prette \& Del Prette, 2001). Desta forma, um bom repertório não somente irá auxiliar no bom convívio com colegas, criando um ambiente saudável de trabalho, mas também na satisfação de trabalhar (Bolsoni-Silva, 2002), na produtividade (Feitoza, Silva, Feitoza, Oliveira, \& Oliveira, 2008) e na prevenção de doenças relacionadas ao trabalho (Bomsucesso, 2002). Como podem ser observadas nos resultados deste estudo, as habilidades sociais tiveram uma associação com a satisfação com o trabalho, visto que o grupo com altas habilidades sociais apresentou média de satisfação no trabalho superior e estatisticamente significativa em relação ao grupo com baixas habilidades sociais.

\section{Considerações Finais}

Este artigo teve como objetivo relacionar o comportamento social (composto pelas habilidades sociais e os pensamentos negativos relacionados às interações) e interações sociais consideradas como fatores de risco ou proteção para o desenvolvimento de adultos emergentes, a partir da comparação de grupos divididos a partir de seus níveis de habilidades sociais e pensamentos negativos. As habilidades sociais apresentaram relações positivas com as interações protetivas e relações negativas com as de risco. Já os pensamentos negativos apresentaram relações positivas com os fatores de risco e relações negativas com os fatores de proteção. Um repertório de habilidades sociais adequado pode auxiliar os indivíduos a lidarem com problemas, bem como a construir relações sociais significativas. Essas relações podem se tornar uma rede de apoio e um fator protetivo, em especial nos casos em que as habilidades sociais não foram suficientes para enfrentarem seus problemas. Por outro lado, a presença de pensamentos negativos pode aumentar a ansiedade nas situações sociais, comprometendo o desempenho social. Este comprometimento tem um impacto negativo na autoestima e na autoeficácia da pessoa. Além disso, pode dificulta o estabelecimento de relações satisfatórias, o que pode resultar em isolamento social e aumentar a chance de transtornos psicológicos.

Em relação às limitações do estudo, salienta-se que se trata de um estudo transversal com medidas de autorrelato. Portanto, as análises utilizadas são apenas comparações entre os grupos com diferentes níveis de habilidades sociais e pensamentos negativos, as quais não são sensíveis o suficiente para avaliar a causalidade de efeito entre as variáveis. Desta forma, é necessária cautela ao realizarem-se generalizações 
destes resultados. Da mesma forma, a amostra foi não apresentou grande variabilidade em relação a algumas variáveis relevantes no contexto brasileiro, como nível socioeconômico e educacional.

Sugere-se que futuras pesquisas estudem as relações entre as habilidades sociais e outros fatores de risco como uso de drogas, além de estudar a relação com outros contextos sociais, como relacionamento conjugal e parental. Além disso, sugere-se a replicação desta pesquisa com amostras clínicas, como usuários de drogas e/ou outros transtornos psicológicos. Estudos longitudinais também seriam ideais para poder avaliar o impacto do repertório de habilidades sociais no decorrer da adultez emergente. Estes estudos poderão auxiliar no entendimento de como as habilidades sociais interagem com estes transtornos; e se elas atuam como um fator de proteção nesta população, da mesma forma que na amostra deste estudo.

\section{Referências}

American Psychiatric Association, APA. (2014). Manual Diagnóstico e Estatístico de Transtornos Mentais - DSM-5 (5 $5^{\mathbf{a}}$ ed.). Porto Alegre: Artmed.

Antunes, R. (2011). Os modos de ser da informalidade: rumo a uma nova era da precarização estrutural do trabalho? Serviço Social \& Sociedade, 107, 405-419. http://dx.doi.org/10.1590/S0101-66282011000300002

Arnett, J. J. (2011). Emerging adulthood(s): The cultural psychology of a new life stage. In J. A. Lene (Ed.), Bridging cultural and developmental approaches to psychology: New synthesis in theory, research, and policy (pp. 255-275). New York, NY: Oxford University Press.

Beesdo-Baum, K., Klotsche, J., Knappe, S., Craske, M. G., LeBeau, R. T., Hoyer, J., Strobel, A., Pieper, L., \& Wittchen, H. (2012). Psychometric properties of the dimensional anxiety scales for DSM-5 in an unselected sample of German treatment seeking patients. Depression and Anxiety, 29(12), 1014-1024. http://dx.doi.org/10.1002/da.21994

Blustein, D. L. (2006). The Psychology of Working: A New Perspective for Career Development, Counseling, and Public Policy. Mahwah, N.J.: Lawrence Erlbaum.

Bolsoni-Silva, A. T. (2002). Habilidades sociais: breve análise da teoria e da prática à luz da análise do comportamento. Interação em Psicologia, 6(2), 233-242. Retrieved from http://ojs.c3sl.ufpr.br/ojs2/index.php/psicologia/article/ view/3311. http://dx.doi.org/10.5380/psi.v6i2.3311

Bomsucesso, E. (2002). Relações interpessoais e qualidade de vida: No trabalho. Rio de Janeiro: Qualitymark.

Caballo, V. E. (2003). Manual de avaliação e treinamento das habilidades sociais. São Paulo: Santos.

Dell'Aglio, D. D., Koller, S. H., Cerqueira-Santos, E., \& Colaço, V. F. R. (2011). Revisando o Questionário da Juventude Brasileira: Uma nova proposta. In D. D. Dell'Aglio \& S. H. Koller (Eds.), Adolescência e juventude: Vulnerabilidade e contextos de proteção (pp. 259-270). São Paulo: Casa do Psicólogo.

Del Prette, A. \& Del Prette, Z. A. P. (2001). Psicologia das relações interpessoais: vivências para o trabalho em grupo. Petrópolis: Vozes.

Del Prette, Z. A. P. \& Del Prette, A. (2009). Psicologia das Habilidades Sociais: diversidade teórica e suas implicações. Petrópolis: Vozes.

Demır, M. \& Weitekamp, L. A. (2007). I am so happy 'cause today I found my friend: friendship and personality as predictors of happiness. Journal of Happiness Studies, 8(2), 181-211. http://dx.doi.org/10.1007/s10902-006-9012-7

DeSousa, D. A., Moreno, A. L., Osório, F. L., Crippa, J. A. S., LeBeau, R., Manfro, G. G., ... Koller, S. H. (2016). Psychometric properties of the dimensional anxiety scales for DSM-5 in a Brazilian community sample. International Journal of Methods in Psychiatric Research. https://doi.org/10.1002/mpr.1531

Donnellan, M. B., Conger, R. D., \& Burzette, R. G. (2007). Personality development from late adolescence to young adulthood: differential stability, normative maturity, and evidence for the maturity-stability hypothesis. Journal of Personality, 75, 237-64. http://dx.doi.org/10.1111/j.1467-6494.2007.00438.x

Dutra-Thomé, L. \& Koller, S. H. (2014a). O significado do trabalho na visão de jovens brasileiros: uma análise de palavras análogas e opostas ao termo "trabalho". Revista Psicologia Organizações e Trabalho, 14(04), $367-380$. Retrieved from: http://pepsic.bvsalud.org/scielo.php?script=sci_arttext\&pid=S1984-66572014000400004

Dutra-Thomé, L., Koller, S. H., (2014b). Emerging adulthood in brazilians of differing socioeconomic status: transition to adulthood. Paidéia (Ribeirão Preto), 24(59), 313-322. http://dx.doi.org/10.1590/1982-43272459201405

Fehr, B. A. (1996). Friendship processes. London: Sage. http://dx.doi.org/10.4135/9781483327440

Feitoza, S., Silva, A. C., Feitoza, A. M., Oliveira, M. M., \& Oliveira, V. M. (2008). O Repertório de habilidades sociais de profissionais que atuam com vendas no setor comercial de tecnologia da informação e suas implicações na qualidade da prestação de serviços. In XXVIII Encontro Nacional de Engenharia de Produção: A integração de cadeias produtivas com a abordagem da manufatura sustentável, Rio de Janeiro, Brasil. Retrieved from: http://www.abepro.org.br/ biblioteca/enegep2008_tn_stp_070_502_11817.pdf 
Hutz, C. S. \& Zanon, C. (2011). Revisão da adaptação, validação e normatização da escala de autoestima de Rosenberg. Avaliação Psicológica, 10(1), 41-49. Retrieved from: http://pepsic.bvsalud.org/scielo.php?script=sci_arttext\& pid=S1677-04712011000100005

LeBeau, R. T., Glenn, D. E., Hanover, L. N., Beesdo-Baum, K., Wittchen, H., \& Craske, M. G. (2012). A dimensional approach to measuring anxiety for DSM-5. International Journal of Methods in Psychiatric Research, 21(4), $258-272$. http://dx.doi.org/10.1002/mpr.1369

Masten, A. S. \& Tellegen, A. (2012). Resilience in developmental psychopathology: contributions of the project competence longitudinal study. Development and Psychopathology, 24, 345-361. http://dx.doi.org/10.1017/ S095457941200003X

Pesce, R. P., Assis, S. G., Santos, N., \& Oliveira, R. V. C., (2004). Risco e proteção: em busca de um equilíbrio promotor de resiliência. Psicologia: Teoria e Pesquisa, 20(2), 135-143. http://dx.doi.org/10.1590/S0102-37722004000200006

Poletto, M. \& Koller, S. H. (2008). Contextos ecológicos: Promotores de resiliência, fatores de risco e proteção. Estudos de Psicologia (Campinas), 25(3), 405-416. http://dx.doi.org/10.1590/S0103-166X2008000300009

Rodrigues, J. S. M. \& Ferreira, N. M. L. A. (2012). Structure and functionality of the social support network for adults with cancer. Acta Paulista de Enfermagem, 25(5), 781-787. http://dx.doi.org/10.1590/S0103-21002012000500021

Sbicigo, J. B., Bandeira, D. R., \& Dell’Aglio, D. D. (2010). Rosenberg Self-Esteem Scale (RSS): factorial validity and internal consistency. Psico-USF, 15(3), 395-403. http://dx.doi.org/10.1590/S1413-82712010000300012

Sbicigo, J. B., Teixeira, M. A. P., Dias, A. C. G., \& Dell'Aglio, D. D. (2012). Propriedades psicométricas da escala de autoeficácia geral percebida (EAGP). Psico, 43(2), 139-146. Retrieved from: http://revistaseletronicas.pucrs.br/ojs/ index.php/revistapsico/article/view/11691

Segrin, C. (2000). Social skills deficits associated with depression. Clinical Psychology Review, 20(3), 379-403. http:// dx.doi.org/10.1016/S0272-7358(98)00104-4

Souza, L. K. de, \& Hutz, C. S. (2007). A qualidade da amizade: adaptação e validação dos questionários McGill. Aletheia, 25, 82-96. Retrieved from: http://pepsic.bvsalud.org/scielo.php?script=sci_arttext\&pid=S1413-03942 007000100007

Swan, J. A., De Moraes, L. F. R., \& Cooper, C. L. (1993). Developing the occupational stress indicator (OSI) for use in Brazil: A report on the reliability and validity of the translated OSI. Stress Medicine, 9(4), 247-253. http://dx.doi org/10.1002/smi.2460090407

Tanner, J. L., \& Arnett, J. J. (2009). The emergence of "emerging adulthood": the new life stage between adolescence and adulthood. In A. Furlong (Ed.), Handbook of youth and young adulthood: new perspectives and agendas (pp. 39-45). London and New York: Routledge.

Wagner, M.F., Pereira, A.S., \& Oliveira, M.S., (2014). Intervención sobre las dimensiones de la ansiedad social por medio de um programa de entrenamiento en habilidades sociales. Psicología Conductual, 22, 423-448. Retrieved from: http://www.funveca.org/revista/pedidos/product.php?id_product=647

\footnotetext{
Autores:

Anderson Siqueira Pereira - Mestre, Universidade Federal do Rio Grande do Sul.

Luciana Dutra Thomé - Doutora, Universidade Federal do Rio Grande do Sul.

Silvia Helena Koller - Doutora, Universidade Federal do Rio Grande do Sul.

Endereço para correspondência:

Anderson Siqueira Pereira

Rua Taquary, 635/102 - Bairro Cristal

90810-180 Porto Alegre, RS, Brasil

<anderson.siqueira.pereira@gmail.com>

Recebido em: 21.03.2016

Aceito em: 13.09.2016
} 